\title{
Analysis of a Repairable System Operating Under Different Weather Conditions
}

\author{
Ashish K. Barak \\ Department of Mathematics \\ Manipal University Jaipur, \\ Jaipur-303007, India
}

\begin{abstract}
The purpose of this paper is to develop and analyze a reliability model of a repairable system of single-unit considering two weather conditions-normal and abnormal. In this model a unit may fail totally either directly from normal mode or via partial failure. There is a single server who visits the system immediately to do repair of the failed unit. The operation and repair of the unit are stopped in abnormal weather as a precautionary measure to avoid excessive damage to the system. Failure rates, repair rates and rate of change of weather conditions follow general distribution. By using semi-Markov process and regenerative point technique some reliability and economic measures of system effectiveness are obtained. The graphical of MTSF, availability and profit with respect to abnormal weather rate has also been shown for a particular case.
\end{abstract}

\section{AMS Subject Classification: 90B25 and 60K10}

\section{Keywords}

Reliability Model, Weather conditions, Repairable System, semi-Markov process and Profit Analysis.

\section{INTRODUCTION}

It is very difficult to keep the environmental conditions under control which may fluctuate due to changing climate, voltage and other natural catastrophic. However, it becomes necessary to protect the operation of the system in abnormal weather for reducing the down time and maintaining the reliability of the system. Generally, the given controlled conditions reckon to normal weather; otherwise, weather is taken as abnormal. A number of reparable systems which operate under strict control of temperature, voltage, storm and moisture, etc. have been considered by the researchers including Osaki (1972), Goyal (1984), Naidu and Gopalan (1984) and Singh (1989) in the field of reliability theory. These conditions when satisfied correspond to normal weather; otherwise, it is supposed that the system is working under abnormal weather. Dhillon and Natesan (1983) and Goel etal.(1985) have studied the stochastic behavior of systems operating under different weather conditions by assuming that repair of the unit is always possible and economical to the system. However, sometimes repair of the unit is not possible and beneficial due to excessive use and high cost of maintenance. In such a situation, the unit may be replaced by new one in order to increase the availability of the system and hence profit. Recently, Chander and Bansal (2005) have discussed singleunit reliability models by introducing the concept of inspection to examine the feasibility of repair.

While incorporating the idea of weather conditions, a singleunit reliability model of a repairable system is analyzed where a unit may fail completely either directly from normal mode or via partial failure. There is a single server who can be made available promptly whenever needed. Server repairs the unit

\author{
M.S. Barak \\ Department of Mathematics, \\ IGPGRC, Rewari, M.D. \\ University, Rohtak-124001, \\ India
}

at its complete failure. The unit after repair works as new. All random variables are mutually independent. The operation and repair of the unit are not permitted in abnormal weather as a precautionary measure to avoid excessive damage to the system. It is assumed that the distribution of failure time and time to change in weather conditions and repair time are general distribution. The system is observed at suitable regenerative epochs by using regenerative point technique. Some economic-related reliability characteristics such as mean sojourn times, mean time to system failure (MTSF), steady state availability, busy period and expected numbers of visits by the server are obtained. Finally, the profit is evaluated for the system to carry out the cost-benefit analysis. Graphs are drawn to show the behavior of MTSF and profit of the model for a particular case when all distributions are taken as negative exponential.

\section{NOTATIONS}

\section{O : Operative state}

E

$\mathrm{f}_{1}(\mathrm{t}) / \mathrm{f}_{2}(\mathrm{t}) / \mathrm{f}(\mathrm{t})$

each model

$\mathrm{F}_{1}(\mathrm{t}) / \mathrm{F}_{2}(\mathrm{t}) / \mathrm{F}(\mathrm{t})$

$\mathrm{pdf} / \mathrm{cdf}$ of failure rate from normal mode to partial failure mode /partial failure mode to total failure mode/normal mode to total failure mode.

$\mathrm{z}(\mathrm{t}) / \mathrm{Z}(\mathrm{t}), \mathrm{z}_{1}(\mathrm{t}) / \mathrm{Z}_{1}(\mathrm{t}): \quad$ p.d.f./c.d.f. of time to change of weather conditions form normal to abnormal, abnormal to normal

$\mathrm{p} / \mathrm{q}$ Probability that repair is not feasible/feasible.

$\mathrm{g}(\mathrm{t}) / \mathrm{G}(\mathrm{t}) \quad$ : $\quad$ pdf/cdf of repair times of completely failed unit

$\mathrm{h}(\mathrm{t}) / \mathrm{H}(\mathrm{t}):$ $\mathrm{O} / \mathrm{PF}$ $\mathrm{pdf} / \mathrm{cdf}$ of inspection time. Unit is operative and in normal mode/unit is partially failed

\section{$\bar{O} / \overline{P F}$}

$\mathrm{FU}_{\mathrm{i}} / \mathrm{FU}_{\mathrm{r}}$

$\overline{F W_{r}} / \overline{F W_{i}}$

$\mathrm{q}_{\mathrm{ijj}}(\mathrm{t}), \mathrm{Q}_{\mathrm{ijj}}(\mathrm{t})$
Unit is good / partially failed but not working due to abnormal weather Unit is totally failed and under inspection/under repair.

\section{: Unit is completely failed and} waiting for repair/ inspection due to abnormal weather. pdf and cdf of direct transition time from a regenerative state $i$ to a regenerative state $\mathrm{j}$ or a failed state $\mathrm{j}$ without visiting any other regenerative state in $(0, \mathrm{t}]$

: cdf of first passage time from regenerative state $i$ to a failed state.

Probability that the system is up at epoch $\mathrm{t} / \mathrm{E}_{0}=\mathrm{S}_{\mathrm{i}} \in \mathrm{E}$

Probability that server is busy in the system at instant $t / E_{0}=S_{i} \in E$. 
$\mathrm{N}_{\mathrm{i}}(\mathrm{t})$

: Expected number of visits by the server in $(0, \mathrm{t}] / \mathrm{E}_{0}=\mathrm{S}_{\mathrm{i}} \in \mathrm{E}$

$\mathrm{M}_{\mathrm{i}}(\mathrm{t}) \quad$ : Probability that system initially in regenerative state $S_{i}$ remains up till time ' $t$ ' without making anymtransition to any other regenerativemstate or returning itself through one ormmore nonregenerative states.

$\mathrm{W}_{\mathrm{i}}(\mathrm{t})$ : Probability that the server is busy in the state $\mathrm{S}_{\mathrm{i}}$ up to time $t$ without making any transition to any other regenerative state or returning tothe same state via one or more non-regenerative state.

$\mathrm{m}_{\mathrm{ij}}$ : Contribution to mean sojourn time in state $S_{\mathrm{i}}$ when system transits directly to state $\mathrm{S}_{\mathrm{j}}\left(\mathrm{S}_{\mathrm{i}}, \mathrm{S}_{\mathrm{j}} \in \mathrm{E}\right)$ so that $\mu_{\mathrm{i}}=\sum_{i} m_{i j}$, where $m_{i j}=\int q_{i j}(t) d t=$

$\int d Q_{i j}(t) d t=-\left[\frac{d}{d s}\left(Q_{i j}^{*}(s)\right]_{s=0}\right.$ and $\mu_{\mathrm{i}}$ is the mean

sojourn time in state $S_{i} \in E$.

(S)(C) $\quad$ symbols for Stieltjes convolution /Laplace convolution.

/*: symbols for Laplace Stieltjes transform(L.S.T.)/Laplace transform L.T

The possible transitions states of system models are respectively shown in the following figure:

Figure 1

$$
\begin{array}{rlr}
p_{1,4} & =\int_{0}^{\infty} z(t) \overline{F_{2}(t)} d t \quad p_{2,0}=\int_{0}^{\infty} g(t) \overline{Z(t)} d t \\
p_{2,5} & =\int_{0}^{\infty} z(t) \overline{G(t)} d t
\end{array}
$$

It can be easily verified that

$$
\begin{aligned}
& p_{30}=p_{52}=p_{41}= \\
& p_{01}+p_{02}+p_{03}=p_{14}+p_{12}=p_{20}+p_{25}=1
\end{aligned}
$$

The mean sojourn times $\mu_{i}$ in the state $S_{i}$ are given by

$$
\begin{array}{ll}
\mu_{0}=\int_{0}^{\infty} \overline{Z(t)} \overline{F_{1}(t)} \overline{F(t)} d t & \mu_{1}=\int_{0}^{\infty} \overline{Z(t)} \overline{F_{2}(t)} d t \\
\mu_{2}=\int_{0}^{\infty} \overline{Z(t) G(t)} d t & \mu_{3}=\int_{0}^{\infty} \overline{Z_{1}(t)} d t \\
\mu_{4}=\int_{0}^{\infty} \overline{Z_{1}(t)} d t & \mu_{5}=\int_{0}^{\infty} \overline{Z_{1}(t)} d t
\end{array}
$$

All the transition states of the model are regenerative.

\section{TRANSITION PROBABILITIES AND MEAN SOJOURN TIMES}

It can be observed that the epoch of entry into any of the states $\mathrm{S}_{\mathrm{i}} \in \mathrm{E}$ are regenerative point. Let $\mathrm{T}_{0}(\equiv 0), \mathrm{T}_{1}, \mathrm{~T}_{2}, \ldots \ldots$. denote the epochs at which the system enters any state $\mathrm{S}_{\mathrm{i}} \in \mathrm{E}$. Let $X_{n}$ denote the state visited at epoch $T_{n}+$, i.e just after transition at $T_{n}$. Then $\left\{X_{n}\right.$, $T_{n}$ \} is a Markov-renewal process with state space $E$ and

$\mathrm{Q}_{\mathrm{ij}}(\mathrm{t})=\operatorname{Pr}\left\{\mathrm{X}_{\mathrm{n}+1}=\mathrm{j}, \mathrm{T}_{\mathrm{n}+1}-\mathrm{T}_{\mathrm{n}} \leq \mathrm{t} \mid \mathrm{X}_{\mathrm{n}}=\mathrm{i}\right\} \quad$ is the semi-Markov kernel over $E$.

The transition probability matrix of embedded Markov-chain is

$\mathrm{p}=\left(\mathrm{p}_{\mathrm{ij}}\right)=\left(\mathrm{Q}_{\mathrm{ij}}(\infty)=\mathrm{Q}(\infty)\right)$ with non-zero elements.

By probabilistic arguments, the non-zero elements $\mathrm{p}_{\mathrm{ij}}$ are

$$
p_{0,1}=\int_{0}^{\infty} f_{1}(t) \overline{Z(t)} \overline{F(t)} d t
$$

Where $\mathrm{p}_{01}$ means that probability of the normal unit is partially failed at time $t$.

All other transition probabilities can be explained in the same manner and given by

$$
\begin{aligned}
& p_{30}=p_{41}=p_{52}=1 \quad p_{0,2}=\int_{0}^{\infty} f(t) \overline{Z(t)} \overline{F_{1}(t)} d t \\
& p_{0,3}=\int_{0}^{\infty} z(t) \overline{F_{1}(t)} \overline{F(t)} d t \quad p_{1}=\int_{0}^{\infty}\left(f \overline{) t_{2}}\right.
\end{aligned}
$$

(3)

$$
\mathrm{m}_{\mathrm{ij}}=\int \mathrm{tdQ}_{\mathrm{ij}}(\mathrm{t})=-\left[\frac{\mathrm{d}}{\mathrm{ds}}\left(\mathrm{Q}_{\mathrm{ij}}^{* *}(\mathrm{~s})\right)\right]_{\mathrm{s}=0}
$$

\section{Relationship between Unconditional Mean and Mean}

\section{Sojourn Times}

$$
\begin{array}{ll}
m_{01}+m_{02}+m_{03}=\mu_{0}, & m_{12}+m_{14}=\mu_{1} \\
m_{20}+m_{25}=\mu_{2}, m_{30}=\mu_{3}, & m_{41}=\mu_{1}, m_{52}=\mu_{5}
\end{array}
$$

\section{RELIABILITY AND MEAN TIME TO SYSTEM FAILURE}

Let $\phi_{i}(t)$ be the c.d.f of first passage time from the regenerative state $i$ to a failed state. Regarding the failed state as absorbing state, we have the following recursive relation for $\phi_{\mathrm{i}}(\mathrm{t})$ :

$$
\phi_{\mathrm{i}}(\mathrm{t})=\sum_{\mathrm{j}} \mathrm{Q}_{\mathrm{i}, \mathrm{j}}(\mathrm{t}) \circledast \phi_{\mathrm{j}}(\mathrm{t})+\sum_{\mathrm{k}} \mathrm{Q}_{\mathrm{i}, \mathrm{k}}(\mathrm{t})
$$

Where $j$ is an un-failed regenerative state to which the given regenerative state $i$ can transit and $k$ is a failed state to which the state $i$ can transit directly.Taking LST of above relations (5) and solving for $\phi_{0}^{* * *}(s)$, we have 
$\mathrm{R}^{*}(\mathrm{~s})=\frac{1-\phi_{0}^{* *}(\mathrm{~s})}{\mathrm{s}}$

The reliability of the system model can be obtained by taking inverse LT of (6) MTSF is given by

$$
\text { MTSF }=\lim _{s \rightarrow 0} R^{*}(s)=\frac{N_{1}}{D_{1}}
$$

where $\mathrm{N}_{1}=$

$$
\begin{aligned}
& m_{03}+m_{30} p_{03}+m_{14} p_{41}+m_{41}+p_{03}^{\prime} p_{14}+p_{30}^{\prime} p_{14} p_{03} \\
& +p_{14}^{\prime} p_{03}+p_{41}^{\prime} p_{14} p_{03}+m_{02}+m_{01} p_{12}+m_{12} p_{01}+p_{02}^{\prime} \\
& p_{14}+p_{14}^{\prime} p_{02}+p_{41}^{\prime} p_{14} p_{02}
\end{aligned}
$$$$
\mathrm{D}_{1}=1-p_{14} p_{41}-p_{03} p_{30}+p_{03} p_{30} p_{41} p_{14}
$$

\section{STEADY STATE AVAILABILITY}

Let $A_{i}(t)$ be the probability that the system is in up state at instant ' $\mathrm{t}$ ' given that the system entered regenerative state $i$ at $t$ $=0$. The recursive relations for $\mathrm{A}_{\mathrm{i}}(\mathrm{t})$ are given as

$A_{i}(t)=M_{i}(t)+\sum_{j} q_{i, j}(t) \subseteq A_{j}(t)$

where $j$ is any successive regenerative state to which the regenerative state $i$ can transit and $\mathrm{M}_{\mathrm{i}}(\mathrm{t})$ 's obtained as

$$
M_{0}=\int_{0}^{\infty} \overline{Z(t)} \overline{F_{1}(t)} \overline{F(t)} d t M_{1}=\int_{0}^{\infty} \overline{Z(t)} \overline{F_{2}(t)} d t
$$

Taking LT of relations (8) and solving for $\mathrm{A}_{0}^{*}(\mathrm{~s})$. The steady state availability can be determined as

$$
A_{0}(\infty)=\lim _{s \rightarrow 0} s A_{0}^{*}(s)=\frac{N_{2}}{D_{2}}
$$

where

$$
\begin{aligned}
N_{2}= & \left(1-p_{25} p_{52}\right)\left[\mu_{0}\left(1-p_{41} p_{14}\right)+p_{01} \mu_{1}\right] \\
D_{2}= & \left(\mu_{0}+p_{03} \mu_{3}\right)\left[\left(1-p_{41} p_{14}\right)\left(1-p_{25} p_{52}\right)\right] \\
& +\left(\mu_{1}+p_{14} \mu_{4}\right)\left[\left(p_{01}\right)\left(1-p_{25} p_{52}\right)\right]+ \\
& \left(\mu_{2}+p_{25} \mu_{5}\right)\left[\left(1-p_{41} p_{14}\right) p_{02}+p_{01} p_{12}\right]
\end{aligned}
$$

\section{BUSY PERIOD ANALYSIS}

Let $\mathrm{B}_{\mathrm{i}}(\mathrm{t})$ be the probability that the server is busy in repairing the unit at an instant ' $t$ ' given that the system entered regenerative state $i$ at $t=0$. The recursive relations for $B_{i}(t)$ are given as

$$
\mathrm{B}_{\mathrm{i}}(\mathrm{t})=\mathrm{W}_{\mathrm{i}}(\mathrm{t})+\sum_{\mathrm{j}} \mathrm{q}_{\mathrm{i}, \mathrm{j}}(\mathrm{t}) \odot \mathrm{B}_{\mathrm{j}}(\mathrm{t})
$$

where $\mathrm{j}$ is any successive regenerative state to which the regenerative state $\mathrm{i}$ can transit and $\mathrm{Wi}(\mathrm{t})$ 's obtained as

$$
W_{2}(t)=\int_{0}^{\infty} \overline{Z(t)} \overline{G(t)} d t
$$

Taking LT of relations (11) and solving for $\mathrm{B}_{0}^{*}(\mathrm{~s})$. The busy period of the server can be determined as.

$B_{0}=\lim _{s \rightarrow 0} s B_{0}^{*}(s)=\frac{N_{3}}{D_{2}}$

Where

$$
\mathrm{N}_{3}=W_{2}\left[p_{01} p_{12}+p_{02}\left(1-p_{41} p_{14}\right)\right]
$$

and D2 has already mentioned.

\section{EXPECTED NUMBER OF VISITS BY THE SERVER}

Let $\mathrm{Ni}(\mathrm{t})$ be the expected number of visits by the server in $(0$, $\mathrm{t}$ ] given that the system entered the regenerative state $\mathrm{i}$ at $\mathrm{t}=0$, we have the following recurrence relations for $\mathrm{Ni}(\mathrm{t})$ :

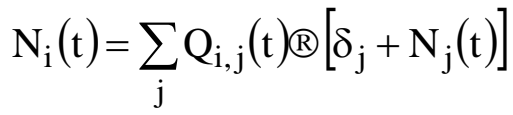

Where $j$ is any regenerative state to which the given regenerative state $i$ transits.

$\delta_{j}=\left\{\begin{array}{ll}1 & j \text { is regenerative state } \\ 0 & \text { otherwise }\end{array}\right\}$

Taking LST of the relation (14) and solving for $\mathrm{N}_{0}^{* *}$ (s). The expression for expected number of visits per unit time is given by

$$
\mathrm{N}_{0}=\lim _{\mathrm{s} \rightarrow 0} \mathrm{~s} \mathrm{~N}_{0}^{* * *}(\mathrm{~s})=\frac{\mathrm{N}_{4}}{\mathrm{D}_{2}}
$$

Where

$\mathrm{N}_{4}=\left[1-p_{25} p_{52}\right]\left[p_{02}\left(1-p_{14} p_{41}\right)+p_{01} p_{12}\right]$

and $\mathrm{D}_{2}$ has already mentioned.

\section{PROFIT ANALYSIS}

Any manufacturing industry is basically a profit making organization and no organization can survive for long without 
minimum financial returns for its investment. There must be an optimal balance between the reliability aspect of a product and its cost. The major factors contributing to the total cost are availability, busy period of server and expected number of visits by the server. The cost of these individual items varies with reliability or mean time to system failure. In order to increase the reliability of the products, we would require a correspondingly high investment in the research and development activities. The production cost also would increase with the requirement of greater reliability.

The revenue and cost function leads to the profit function of a firm/organization, as the profit is excess of revenue over the cost of production. The profit function in time $t$ is given by

$\mathrm{P}(\mathrm{t})=$ Expected revenue in $(0, \mathrm{t}]-$ Expected total cost in $(0, \mathrm{t}]$

In general, the optimal policies can more easily be derived for an infinite time span or compared to a finite time span. The profit per unit time, in infinite time span is expressed as

$$
\lim _{t \rightarrow \infty} \frac{P(t)}{t}
$$

i.e. profit per unit time $=$ total revenue per unit time - total cost per unit time. Considering the various costs, the profit equation is given as

$\mathrm{P}=\mathrm{K}_{0} \mathrm{~A}_{0}-\mathrm{K}_{1} \mathrm{~B}_{0}-\mathrm{K}_{2} \mathrm{~N}_{0}$

Where

$\mathrm{P}=$ Profit per unit time incurred to the system

$\mathrm{K}_{0}=$ Revenue per unit up time of the system

$\mathrm{A}_{0}=$ Total fraction of time for which the system is operative

$\mathrm{K}_{1}=$ Cost per unit time for which server is busy

$\mathrm{B}_{0}=$ Total fraction of time for which the server is busy

$\mathrm{K}_{2}=$ Cost per visit by the server

$\mathrm{N}_{0}=$ Expected number of visits per unit time for the server

\section{RESULTS AND DISCUSSION}

To show the importance of results and characterize the behavior of MTSF and profit of the system, here we assume that failure times of unit, time of change of weather conditions and repair times of the unit as Weibull distributed with two parameters. Probability density function and figure of cumulative distribution function of Weibull distribution with two parameters is given by

$$
h(t)=\lambda t \exp \left[-\lambda t^{b+1} / b+1\right] \quad \mathrm{t} \geq 0
$$

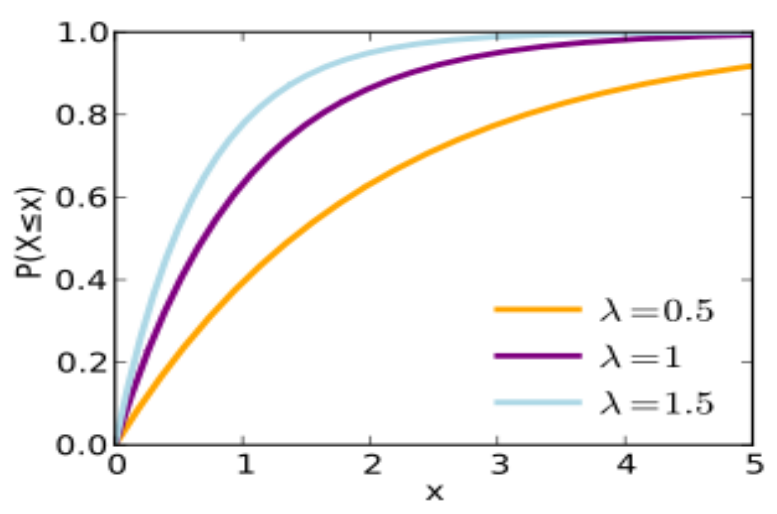

Fig. 2: Cumulative Distribution Function

Where $b$ and $\lambda$ are positive constants and are known as shape and scale parameters respectively. From the properties of Weibull distribution, If $b=0$, it become the exponential distribution.

Let

$$
\begin{aligned}
& f_{1}(t)=\lambda_{1} \exp \left[-\lambda_{1} t^{b+1} / b+1\right] \\
& f_{2}(t)=\lambda_{2} \exp \left[-\lambda_{2} t^{b+1} / b+1\right] \\
& f(t)=\lambda \exp \left[-\lambda t^{b+1} / b+1\right] \\
& \mathrm{z}(\mathrm{t})=\beta \exp \left[-\beta \mathrm{t}^{\mathrm{b}+1} / \mathrm{b}+1\right] \\
& \mathrm{z}_{1}(\mathrm{t})=\beta_{1} \exp \left[-\beta_{1} \mathrm{t}^{\mathrm{b}+1} / \mathrm{b}+1\right] \\
& g(t)=\alpha \exp \left[-\alpha t^{b+1} / b+1\right]
\end{aligned}
$$

For particular values to various parameters and costs, the graphical results for MTSF and profit function are obtained by considering exponential distributions for all random variables associated with failure, weather conditions and repair times as shown in fig 3 and 4.

\section{CONCLUSION}

The graphical behavior of mean time to system failure (MTSF) with respect to abnormal weather rate $(\beta)$ shown in fig.3. It is observed that MTSF increase with the increase of $\beta$. It is also observed that MTSF decreases as direct failure rate $(\lambda)$ and normal weather rate $(\beta 1)$ increase. Thus, we can say that life time of the system keeps on increasing with the increase of abnormal weather $\operatorname{rate}(\beta)$ due to the increase of non working period of the system. Figure 4 highlight the behavior of profit of the model with respect to abnormal weather rate $(\beta)$. It can be seen that profit of the system decreases with the increase of abnormal weather rate $(\beta)$. Further, when failure rate $(\lambda)$ and normal weather rate $(\beta 1)$ increase, the profit of the system increase.

\section{REFERENCES}

[1] Chander, S., Bansal, R.K.,2005. Profit analysis of single -unit reliability models with repair at different failure modes. Proc. INCRESE 2005, IIT Khargpur (India),577588. 
[2] Dhillon, B.S., Natesan, J.,1983. Stochastic analysis of out door power system in fluctuating environment. Microelectron. Reliab.23,867-881.

[3] Goyal, V.,1984. Cost analysis in a two-unit standby system with a regular repairman and patience time. Microelectron. Reliab., 24(3), 453-459.

[4] Goyal, L.R., Gupta. Rakesh , Rastogi, A.K.,1985. Cost analysis of a system with partial failure mode and abnormal weather condition. Microelectron. Reliab.25,461-466.

[5] Naidu, R.S., Gopalan, M.N.,1984. Cost benefit analysisof a one server two-unit system to arbitrary failure, Inspection and repair. Reliability Engg. , 8, 11-22.

[6] Osaki, S.,1972. Reliability analysis of a two-unit standby redundant system with preventive maintenance. IEEE Trans. Reliab., $R-21$.

[7] Singh, S.K.,1989. Profit evaluation of a two unit cold standby system with random appearance and disappearance time of the service facility. Microelectron.Reliab.,29,705-709.

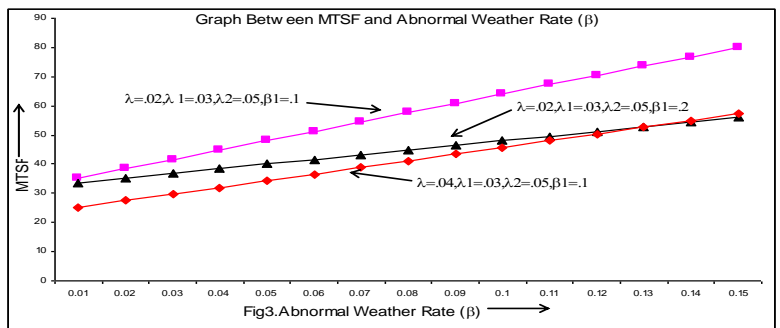

Fig. 3: MTSF Vs. Abnormal Weather Rate

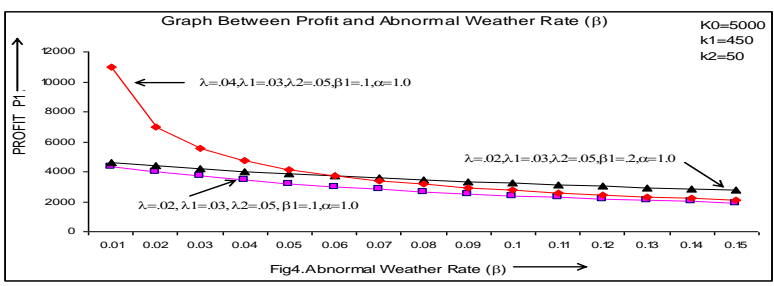

Fig. 4: Profit Vs. Abnormal Weather Rate 